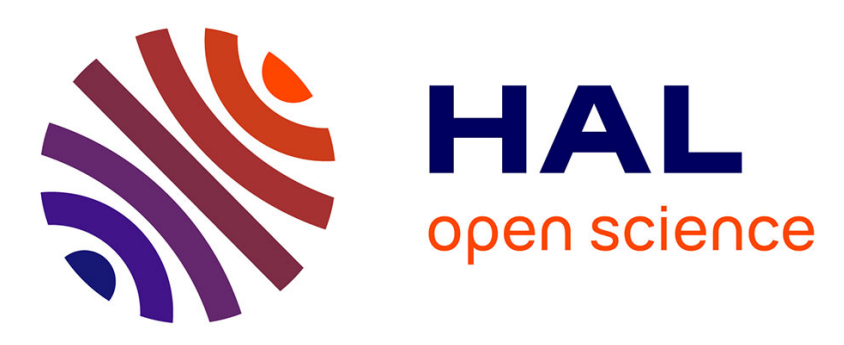

\title{
Analysis of cloaking metasurface using theory of characteristic modes
}

Ozuem Chukwuka, Divitha Seetharamdoo, Mohammed Kalaagi

\section{To cite this version:}

Ozuem Chukwuka, Divitha Seetharamdoo, Mohammed Kalaagi. Analysis of cloaking metasurface using theory of characteristic modes. International Workshop on Antenna Technology (IWAT 2020), Feb 2020, Bucarest, Romania. 10.1109/iWAT48004.2020.1570598014 . hal-02925139v2

\section{HAL Id: hal-02925139 \\ https://hal.science/hal-02925139v2}

Submitted on 6 May 2021

HAL is a multi-disciplinary open access archive for the deposit and dissemination of scientific research documents, whether they are published or not. The documents may come from teaching and research institutions in France or abroad, or from public or private research centers.
L'archive ouverte pluridisciplinaire HAL, est destinée au dépôt et à la diffusion de documents scientifiques de niveau recherche, publiés ou non, émanant des établissements d'enseignement et de recherche français ou étrangers, des laboratoires publics ou privés. 


\title{
Analysis of cloaking metasurface using theory of characteristic modes
}

\author{
Chukwuka Ozuem, Divitha Seetharamdoo and Mohammed Kalaagi \\ Univ. Lille Nord de France, IFSTTAR, COSYS, LEOST \\ F-59650 Villeneuve D’Ascq, France. \\ ozuem.chukwuka@ifsttar.fr, divitha.seetharamdoo@ifsttar.fr, mohammed.kalaagi@ifsttar.fr
}

\begin{abstract}
Metasurfaces are used to design mantle cloaks based on scattering cancellation which involves surface impedance analysis. Theory of characteristic modes is a good tool for analyzing the scattering of a metallic object because it is based on the surface impedance and brings physical insight into the scattering properties of a structure independently of an excitation source. The theory of characteristic modes is applied in the analysis of a cylindrical cloaking metasurface used for a UMTS antenna. The result provides physical insight into the design of mantle cloaks. It shows that the direction of the fundamental modal current distribution of the cloak is at $90^{\circ}$ to that of the UMTS antenna.
\end{abstract}

Index Terms-metasurface unit cell, mantle cloaking, theory of characteristic modes, scattering cancellation

\section{INTRODUCTION}

The concept of electromagnetic cloaking has gained more interest since the realization of metamaterials. It involves controlling the scattering of an object by reducing the scattering cross section (SCS) which makes them invisible to an incoming electromagnetic wave [1][2]. Thus, electromagnetic cloaking has found important application in antenna environment making them less visible to nearby scatters in their surroundings [3].

Among the cloaking techniques of transmission-line network [4], transformation optics [5] and scattering cancellation [6][7], the scattering cancellation also known as mantle cloaking is more desired despite its limitation to the size of the object that can be cloaked. This is because of its easy implementation, available analytical model and its thin thickness. One of the challenge of designing a mantle cloak is finding the optimal parameter of the unit cell which determines the cloak performance however, in [8] it was shown that by properly tailoring the surface reactance of the unit cell, one can achieve reduction on the total scattering of an object. Alessio Monti and co. [9] designed a mantle cloak for co-site radio frequency antenna based on the analytical formulation in $[8][10]$.

Most mantle cloak designs are based on the available analytical models however, considering that the scattering cancellation technique is based on the surface impedance of the structure and the mantle cloak, the theory of characteristic modes (TCM) is suited for the analysis and design of mantle cloaks. TCM is the representation of the current distribution supported on a radiating surface which is derived from the discretization of the surface impedance of the structure. In
[11], numerical analysis based on characteristic mode was done for unit cells of metal infinite arrays where only planar structures were considered.

The aim of this work is to demonstrate the physical properties of a cloaking structure using TCM which would help in developing TCM based design principle for cloaks. In this work a cylindrical cloaking metasurface used for the design of mantle cloak is analyzed using TCM. To allow for comparison of the result and analysis, we consider the cloak used in [9] for cloaking an LTE antenna from interfering with a UMTS antenna when both are co-located in the same site. The result shows that by considering the physical insight gotten from the modal analysis and modal current distribution, a mantle cloak can be easily designed and optimized. The higher order mode is exploited for resonance and the fundamental modal current distribution on the mantle cloak has a direction of $90^{\circ}$ to that of the antenna. TCM is therefore a good tool for designing mantle cloak while having physical insight on the radiation properties of the cloak.

\section{THEORY OF CHARACTERISTIC MODES}

Theory of characteristic modes are a set of orthogonal surface current and radiated field which are supported by a structure. It shows the numerical representation of the current modes supported on a structure and provides physical insight into the radiating properties of the structure [12] with few modes sufficient to represent the global behaviour of the structure [13]. The structure is usually represented by its surface impedance $\mathrm{Z}$ given as:

$$
\mathrm{Z}=\mathrm{R}+\mathrm{j} \mathrm{X}
$$

where $\mathrm{R}$ is the real part and $\mathrm{X}$ is the imaginary part of the impedance. The impedance $\mathrm{Z}$, is used in solving the generalized eigenvalue equation given in equation (2) as:

$$
[\mathrm{X}][\mathrm{I}]=\lambda_{\mathrm{n}}[\mathrm{R}][\mathrm{I}],
$$

where $\lambda_{\mathrm{n}}$ is the eigenvalue of each $\mathrm{n}$ mode and $\mathrm{I}$ is the eigencurrent.

The $\lambda_{\mathrm{n}}$ value can be large and ranges from $-\infty$ to $+\infty$ hence, to get an intuitive knowledge into the behaviour and resonance of the structure, the characteristic angle $\alpha_{n}$ which ranges between $90^{\circ}$ and $270^{\circ}$ is used. $\alpha_{n}$ is derived directly from $\lambda_{\mathrm{n}}$ as:

$$
\alpha_{n}=180^{\circ}-\tan ^{-1} \lambda_{n}
$$


The value of $\lambda_{\mathrm{n}}$ and $\alpha_{n}$ provides information on the modal behaviour of the structure such that with $\lambda_{\mathrm{n}}$ greater than zero and $\alpha_{n}$ less than $180^{\circ}$ the mode is magnetic and when $\lambda_{\mathrm{n}}$ is less than zero and $\alpha_{n}$ is greater than $180^{\circ}$ the mode is electric. A resonant mode has $\lambda_{\mathrm{n}}$ equal to zero and $\alpha_{n}$ equal to $180^{\circ}$. The first resonant mode is referred to as the fundamental resonant mode while the other modes are considered higher order modes.

\section{ANALYSIS OF THE UMTS ANTENNA}

The UMTS antenna as presented in [9] is a monopole antenna operating within the frequency range of $1900 \mathrm{MHz}$ and $2200 \mathrm{MHz}$. The antenna is shown in fig. 1:

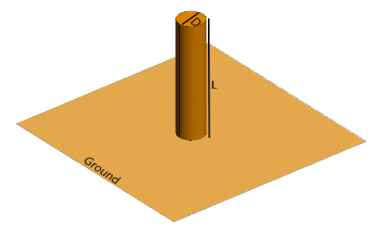

Fig. 1: UMTS monopole antenna.

The length and diameter of the monopole is $\mathrm{L}=35 \mathrm{~mm}$ and $\mathrm{D}=3 \mathrm{~mm}$ respectively. The ground plane used in this analysis is set to an infinite ground plane because a finite ground plane will be seen as a rectangular plate and will add more complexity to the analysis. The TCM analysis is carried out. The eigenvalue curve against frequency and characteristic angle curve against frequency of the first three modes is displayed in fig. 2 and fig. 3 respectively.

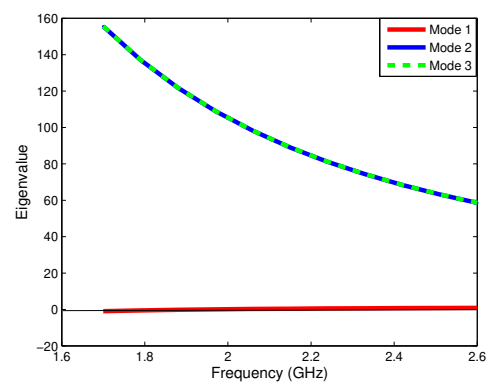

Fig. 2: Eigenvalue against frequency of UMTS monopole antenna.

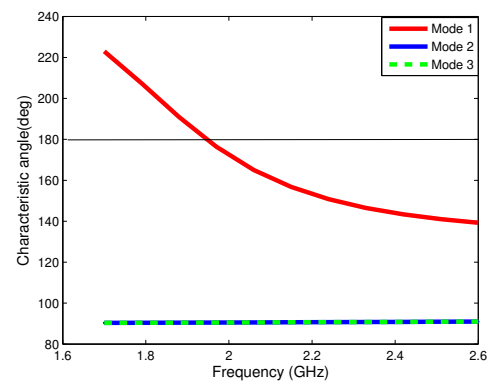

Fig. 3: Characteristic angle against frequency of UMTS monopole antenna.
The curves in fig. 2 and fig. 3 shows that the higher modes, mode 2 in blue and mode 3 in green are magnetic modes. They have similar behaviour since they have the same value throughout the considered frequency band of $1.6 \mathrm{GHz}$ to 2.6 GHz. The fundamental mode, mode 1 in red is a resonating mode and crosses the zero eigenvalue and $180^{\circ}$ characteristic angle at $1.95 \mathrm{GHz}$. Thus, mode 1 is the resonating mode while mode 2 and mode 3 are higher order non-radiating modes.

To get further physical insight into the behaviour of the UMTS monopole structure, we examine the modal surface current distribution of the first three modes shown in fig. 4.
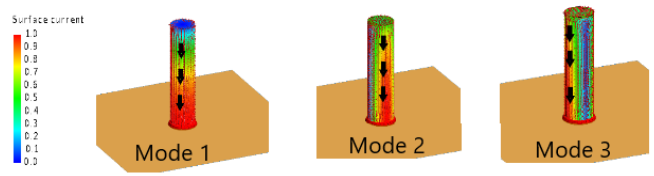

Fig. 4: Normalized modal surface current distribution of UMTS monopole antenna.

The normalized modal surface current distribution shows mode 1 with an electric profile having the flow in only one direction towards the ground. The current density for mode 1 is the same around the cylinder and increases along the length of the cylinder as it approaches the ground plane. Mode 2 and mode 3 have similar current profile with a loop like current around the cylinder. There is a $90^{\circ}$ shift in the position of the maximum and minimum current density between mode 2 and mode 3 .

\section{ANALYSIS OF THE MANTLE CLOAK}

The design of the mantle cloak should be such that it is able to cancel the scattering from the UMTS monopole antenna. In [14], it was shown that by exploiting the resonance of higher order modes, maximum scattering can be achieved. As a result of the challenges associated with TCM analysis of di-electrics such as numerical and modal tracking complexities [12], only the metallic part of the mantle cloak is considered by analysing the metallic part of a unit cell of the cloak. The unit cell of the cylindrical cloak implemented in [9] is shown in fig. 5:

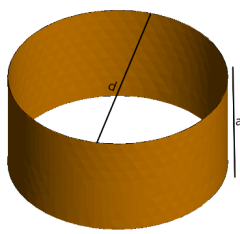

Fig. 5: Cylindrical mantle cloak.

The length and diameter of the cloak is a $=24.07 \mathrm{~mm}$ and $\mathrm{d}=15 \mathrm{~mm}$ respectively. The TCM analysis is carried out. The result represented by the eigenvalue curve against frequency and characteristic angle curve against frequency of the first three modes is shown in fig. 6 and fig. 7 respectively.

The curves in fig. 6 and fig. 7 shows that the mode 3 in green is a magnetic mode while mode 1 in red and mode 


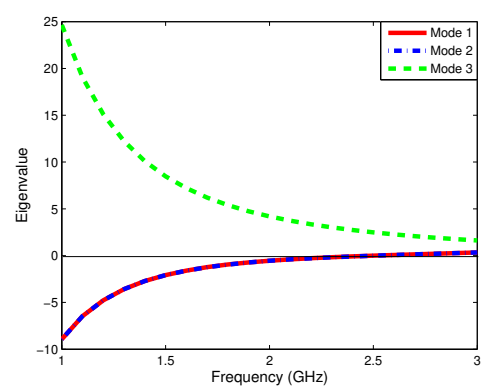

Fig. 6: Eigenvalue against frequency of cylindrical mantle cloak unit cell.

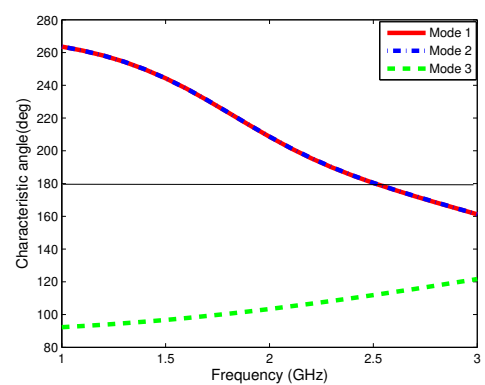

Fig. 7: Characteristic angle against frequency of cylindrical mantle cloak unit cell.

2 in blue have the same behaviour with overlapping values and are resonating modes since they cross the zero eigenvalue and $180^{\circ}$ characteristic angle at $2.51 \mathrm{GHz}$. Mode 1 is the fundamental resonating mode, mode 2 is the resonating higher order mode while mode 3 is the non-resonating higher order mode. As suggested in [14], the higher order resonance of mode 2 is exploited to coincides with the resonance of mode 1 which enhances the absorption properties of the unit cell. The resonating mode 1 and mode 2 of the cloak cancels out the effect of the non-resonating mode 2 and mode 3 of the UMTS monopole antenna. The inclusion of the di-electric substrate would shift the resonance frequency (i.e. frequency at which the eigenvalue and characteristic angle would cross zero and $180^{\circ}$ respectively) to a lower frequency value while retaining the behaviour of the mantle cloak.

To get further physical insight into the behaviour of the mantle cloak structure, we examine the modal surface current distribution of the first three modes as shown in fig. 8:

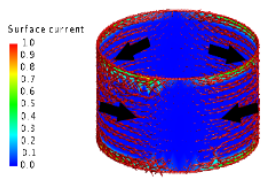

Mode 1

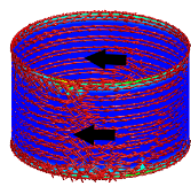

Mode 2

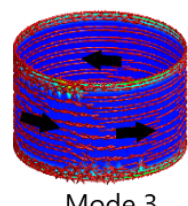

Mode 3
Fig. 8: Normalized modal surface current distribution of cylindrical mantle cloak unit cell.

The normalized modal surface current distribution of the mode 3 shows a magnetic profile with the current flowing around the cylinder in a loop manner. In mode 1 and mode
2 , the current profile are similar with $90^{\circ}$ shift in the position of the maximum and minimum current density. Mode 1 and mode 2 current profile are the same as a dipole current profile which is electric.

From the physical perspective, it is worth noting that the surface current distribution of the non-resonant modes of the UMTS monopole antenna have magnetic current distribution and an inductive behaviour while the resonant modes of the mantle cloak have electric current distribution profile and a capacitive behaviour. There is also a $90^{\circ}$ shift in the direction of their flow i.e. while the current on the UMTS monopole flows in the vertical direction, that of the mantle cloak flows in the horizontal direction. An alternative explanation to the function of the mantle cloak can be the compensation of the inductive behaviour of the non-resonant modes of the UMTS monopole antenna by the capacitive behaviour of the resonant modes of the cloak. The result of the effect of the mantle cloak on the antenna performance is not reproduced here since it can be found in literature [9].

Although only the analysis of a unit cell of the cloak structure was shown, the analysis of the full cloak structure as presented in [9] shows that the physical behaviour in terms of the eigenvalue, characteristic angle and modal surface current distribution are almost the same however, there is a shift in the resonances of the full cloak structure.

\section{CONCLUSION}

The design of a mantle cloak for a UMTS monopole antenna has been analyzed using the TCM numerical tool. By exploiting the resonance of a higher order mode of a unit cell such that it coincides with the fundamental resonance, the absorption properties of the unit cell is maximized. The modal surface current distribution of the first two months for both the antenna and the cloak have a $90^{\circ}$ shift in the direction of the flow. This analysis can help guide the design principle of mantle cloaks while having insight into the radiating properties of the cloak.

\section{ACKNOWLEDGMENT}

The authors acknowledge funding by IFSTTAR for Ph.D. scholarship and SMARTIES in the framework of the ELSAT 2020 program co-financed by the European Union with the European Regional development fund, the French state and Hauts de France Regional council.

\section{REFERENCES}

[1] A. Monti, J. Soric, A. Alù, A. Toscano, and F. Bilotti, "Design of cloaked yagi-uda antennas," EPJ Applied Metamaterials, vol. 3, p. 10, 2016.

[2] A. Monti, M. Barbuto, A. Alù, A. Toscano, and F. Bilotti, "Electromagnetic cloaking for antenna arrays," in 2018 IEEE International Symposium on Antennas and Propagation \& USNC/URSI National Radio Science Meeting. IEEE, 2018, pp. 909-910.

[3] T. V. Teperik and A. de Lustrac, "Electromagnetic cloak to restore the antenna radiation patterns affected by nearby scatter," AIP Advances, vol. 5, no. 12, p. 127225, 2015.

[4] P. Alitalo, F. Bongard, J.-F. Zürcher, J. Mosig, and S. Tretyakov, "Experimental verification of broadband cloaking using a volumetric cloak composed of periodically stacked cylindrical transmission-line networks," Applied Physics Letters, vol. 94, no. 1, p. 014103, 2009. 
[5] W. X. Jiang, T. J. Cui, X. M. Yang, Q. Cheng, R. Liu, and D. R. Smith, "Invisibility cloak without singularity," Applied Physics Letters, vol. 93, no. 19, p. 194102, 2008

[6] P.-Y. Chen and A. Alu, "Mantle cloaking using thin patterned metasurfaces," physical review B, vol. 84, no. 20, p. 205110, 2011.

[7] A. Alù, "Mantle cloak: Invisibility induced by a surface," physical review $B$, vol. 80 , no. 24 , p. $245115,2009$.

[8] Y. R. Padooru, A. B. Yakovlev, P.-Y. Chen, and A. Alù, "Analytical modeling of conformal mantle cloaks for cylindrical objects using subwavelength printed and slotted arrays," Journal of Applied Physics, vol. 112, no. 3, p. 034907, 2012.

[9] A. Monti, J. Soric, M. Barbuto, D. Ramaccia, S. Vellucci, F. Trotta, A. Alù, A. Toscano, and F. Bilotti, "Mantle cloaking for co-site radiofrequency antennas," Applied Physics Letters, vol. 108, no. 11, p. $113502,2016$.

[10] A. Monti, J. C. Soric, A. Alù, A. Toscano, and F. Bilotti, "Anisotropic mantle cloaks for tm and te scattering reduction," IEEE Transactions on Antennas and Propagation, vol. 63, no. 4, pp. 1775-1788, 2015.

[11] Y. Haykir and O. A. Civi, "Characteristic mode analysis of unit cells of metal-only infinite arrays," Advanced Electromagnetics, vol. 8, no. 2, pp. 134-142, 2019.

[12] Y. Chen and C.-F. Wang, Characteristic modes: Theory and applications in antenna engineering. John Wiley \& Sons, 2015

[13] M. Cabedo-Fabres, E. Antonino-Daviu, A. Valero-Nogueira, and M. F. Bataller, "The theory of characteristic modes revisited: A contribution to the design of antennas for modern applications," IEEE Antennas and Propagation Magazine, vol. 49, no. 5, pp. 52-68, 2007.

[14] Y. R. Padooru, A. B. Yakovlev, P.-Y. Chen, and A. Alù, "Line-source excitation of realistic conformal metasurface cloaks," Journal of Applied Physics, vol. 112, no. 10, p. 104902, 2012. 